\title{
Safety of TNF inhibitors in adolescents and children
}

This article was published in the following Dove Press journal:

Adolescent Health, Medicine and Therapeutics

23 December 2010

Number of times this article has been viewed

\section{Lauren Keyser McCluggage}

Clinical Pharmacy, Philadelphia

College of Pharmacy, Philadelphia, PA, USA

Correspondence: Lauren McCluggage

Clinical Pharmacy, Philadelphia College of Pharmacy, 600 South 43rd Street, Box 34,

Philadelphia, PA 19104, USA

$\mathrm{Tel}+\mathrm{I}$ 2/5-596-8745

Fax + I 2I5-596-8586

Email l.mccluggage@usp.edu
Abstract: This article describes the use of tumor necrosis factor (TNF) inhibitors in children, reviews the pharmacology of these agents, and reviews and summarizes the current safety information available for etanercept, adalimumab, and infliximab. TNF inhibitors are being used for a variety of indications in children including Crohn's disease and juvenile idiopathic arthritis. However, the full safety profile of these agents is still not known. In adult patients, TNF inhibitors have demonstrated a variety of adverse effects including increased risk of infection, malignancy, demyelinating disorders, and reactivation of latent diseases. In children the rate of adverse effects is harder to elucidate due to the limited number of patients in clinical trials and limited case reports. However, based on the data available, TNF inhibitors have been implicated in increasing the rate of malignancy in children, especially the rate of lymphoma. In addition, similarly with adults the rate of infections is increased and the types of infections are more rare or opportunistic. One of the more common adverse effects continues to be infusion or injection-site reactions, although children tend to have a lower rate of infusion reactions with infliximab compared to adults. Based on the limited safety information and long-term effect data, TNF inhibitors should be reserved in children for patients with refractory disease and the risks need to be understood and assessed prior to initiation.

Keywords: TNF-alpha inhibitors, adverse effects, safety, children

\section{Introduction}

Tumor necrosis factor (TNF) inhibitors have been used for many autoimmune diseases in both children and adults. These agents have been shown to be efficacious for rheumatoid arthritis, Crohn's disease, ankylosing spondylitis, juvenile idiopathic arthritis (JIA), and psoriatic arthritis and are continually being researched for roles in other disease processes. ${ }^{1-5}$ However, with all medications the risks and benefits must be assessed and balanced.

The TNF inhibitors have been associated with various adverse effects including infections and malignancies. The risk for infection can range from mild infection to reactivation of latent infections such as tuberculosis or hepatitis. In addition, there are less data available on how TNF inhibitors affect children and the risks they pose to this subpopulation. Therefore, the objective of this article is to review the use of TNF inhibitors in children and provide a detailed analysis of the safety information available for these agents, focusing on the agents the US Food and Drug Administration (FDA) has approved for children. 


\section{TNF inhibitor pharmacology}

TNF-alpha exerts a variety of functions within the body. At low concentrations, TNF-alpha induces the body's immune response to local injury or infection. However, in states of overproduction TNF-alpha leads to increased inflammation and possible organ injury. TNF-alpha is overproduced in multiple autoimmune conditions including rheumatoid arthritis, Crohn's disease, psoriasis, psoriatic arthritis, and ankylosing spondylitis. ${ }^{6}$

TNF-alpha is produced from various cells including macrophages, T-cells, mast cells, granulocytes, and fibroblasts. Once released, TNF-alpha exerts many effects depending on the location and underlying medical condition. For example, in patients with rheumatoid arthritis, TNF-alpha initiates matrix degradation and stimulates osteoclast activity, whereas in patients with Crohn's disease, TNF-alpha activity results in granuloma formation. Regardless of the underlying condition, TNF-alpha induces inflammation by stimulation of pro-inflammatory cytokines and is an important component in both innate and adaptive immunity. ${ }^{6}$

Although all of the current TNF inhibitors result in decreasing concentrations of TNF-alpha, there are many differences between them. Infliximab, adalimumab, and golimumab are monoclonal antibodies specific to TNF., ${ }^{1,4-6}$ However, infliximab is a chimeric mouse-human monoclonal antibody which is only $70 \%$ to $75 \%$ humanized whereas adalimumab and golimumab are $100 \%$ humanized. ${ }^{6,7}$ In contrast, etanercept is a dimer of 2 human TNF receptors bound to the Fc portion of a human monoclonal antibody. 3,6 Certolizumab pegol is a humanized antibody Fab fragment and is conjugated to polyethylene glycol., ${ }^{2,6}$

These structural differences result in minor differences in their effects. Infliximab, adalimumab, golimumab, and certolizumab pegol are able to bind both soluble and transmembrane bound TNF-alpha. Etanercept can only bind to soluble TNF-alpha but is able to also bind to TNF-beta which the others cannot. Also, infliximab, adalimumab, and golimumab can induce apoptosis of cells that produce TNF-alpha, but etanercept and certolizumab pegol cannot. Although these differences have been elucidated, the full impact on clinical outcomes has yet to be determined. ${ }^{6}$

The variations among the TNF inhibitors become more apparent when assessing the dosing regimens of the various agents. All except infliximab, which is an intravenous infusion, are given as a subcutaneous injection but with varying intervals ranging from biweekly to monthly. Table 1 outlines some of the key similarities and differences in the pharmacology of the TNF inhibitors.

\section{Clinical efficacy in children}

Of the 5 available TNF inhibitors, only infliximab, adalimumab, and etanercept have FDA-approved indications for children. Infliximab is approved to treat moderately to severely active Crohn's disease in children greater than 6 years old who have not responded adequately to conventional therapy. ${ }^{5}$ Adalimumab is approved to treat moderately

Table I Summary of select pharmacologic properties of tumor necrosis factor-alpha inhibitors

\begin{tabular}{|c|c|c|c|c|c|}
\hline & Adalimumab & Certolizumab & Etanercept & Golimumab & Infliximab \\
\hline Brand name & Humira & Cimzia & Enbrel & Simponi & Remicade \\
\hline FDA-approved & Rheumatoid arthritis & Crohn's disease & Rheumatoid arthritis & Rheumatoid arthritis & Crohn's disease \\
\hline \multirow[t]{5}{*}{ adult indications } & Psoriatic arthritis & Rheumatoid arthritis & Psoriatic arthritis & Psoriatic arthritis & Ulcerative colitis \\
\hline & Ankylosing spondylitis & & Ankylosing spondylitis & Ankylosing spondylitis & Rheumatoid arthritis \\
\hline & Crohn's disease & & Plaque psoriasis & & Ankylosing spondylitis \\
\hline & Plaque psoriasis & & & & Psoriatic arthritis \\
\hline & & & & & Plaque psoriasis \\
\hline FDA indication & $\geq 4$ years old for & & $\geq 2$ years old for & None & $\geq 6$ years old for \\
\hline \multirow[t]{2}{*}{ for children } & polyarticular juvenile & & polyarticular juvenile & & Crohn's disease \\
\hline & idiopathic arthritis & & idiopathic arthritis & & \\
\hline Dosage for & $<15$ kg: not & & $0.4 \mathrm{mg} / \mathrm{kg}$ & & $5 \mathrm{mg} / \mathrm{kg}$ IV weeks 0,2 , \\
\hline children based & recommended & & subcutaneously twice & & and 6 then every 8 \\
\hline \multirow[t]{4}{*}{ on FDA approval } & 15 to $<30 \mathrm{~kg}: 20 \mathrm{mg}$ & & weekly or & & weeks thereafter \\
\hline & every other week & & $0.8 \mathrm{mg} / \mathrm{kg}$ subcutaneously & & \\
\hline & $\geq 30 \mathrm{~kg}: 40 \mathrm{mg}$ every & & weekly (max of & & \\
\hline & other week & & 50 mg per week) & & \\
\hline Class & $100 \%$ humanized & $100 \%$ humanized & Fc portion of $100 \%$ & $100 \%$ humanized & Chimeric monoclonal \\
\hline & monoclonal antibody & $\begin{array}{l}\text { monoclonal antibody } \\
\text { fragment }\end{array}$ & $\begin{array}{l}\text { humanized monoclonal } \\
\text { antibody }\end{array}$ & monoclonal antibody & antibody \\
\hline Half-life & 10-20 days & 14 days & $72-132$ hours & 14 days & $7-12$ days \\
\hline
\end{tabular}


to severely active polyarticular JIA in children 4 years of age or older and etanercept is approved for moderately to severely active polyarticular JIA in children 2 years of age or older. ${ }^{1,3}$ Golimumab and certolizumab have not been shown to be ineffective in children, there are just not any data currently to support their use in children. Therefore, this article will detail the information available for the pediatric-approved medications.

Infliximab was studied in pediatric patients with Crohn's disease as induction therapy and maintenance therapy. After induction therapy (infusions at weeks 0,2 , and 6), 88\% of patients had a clinical response and 59\% were in clinical remission. After 1 year of maintenance therapy, infliximab every 8 weeks, $56 \%$ of patients were in clinical remission. ${ }^{5}$

The landmark study evaluating the effect of etanercept for JIA was conducted in 69 children aged 4 to 17 years old with active polyarticular JIA. All patients had to have been treated with methotrexate with an inadequate response. There were 2 portions to the trial: open label treatment for 3 months; and double blind randomized treatment for months 4 to 7 . In the open label portion of the trial, $74 \%$ of patients treated with etanercept experienced a clinical response defined as $\geq 30 \%$ improvement in 3 of the 6 components (active joint count, number of joints with limitation of motion, physician and patient/parent global assessments, functional assessment, and erythrocyte sedimentation rate). In addition, $64 \%$ experienced a $50 \%$ improvement and $36 \%$ experienced a $70 \%$ improvement. For the double blind portion, only $24 \%$ of patients treated with etanercept experienced a disease flare compared to $77 \%$ of patients treated with placebo $(P=0.003) .{ }^{8}$ In an extension analysis of these patients, etanercept response was durable over 8 years of treatment. ${ }^{9}$

Adalimumab was assessed in a similar manner as etanercept in pediatric patients with JIA. One hundred seventy-one patients with active JIA who either were naïve to methotrexate or previously treated with methotrexate were assessed. During the open label portion of the trial, $84 \%$ of patients treated with adalimumab experienced a response (defined the same as in the etanercept trial). In the double blind portion of the trial, patients treated with adalimumab had a lower rate of disease flare (40\%) compared to patients treated with placebo (68\%). Also of importance is the fact that the patient's or parent's assessment of pain decreased by $48 \%$ to $60 \%$ when adalimumab therapy was initiated. The benefits of adalimumab were similar regardless of previous methotrexate use. ${ }^{10}$

As is apparent from these data, the TNF inhibitors are potent, effective agents that can induce remission and response in patients either naïve or intolerant to more traditional therapies. However whenever assessing medications' benefits, the risks also need to be assessed.

\section{Safety and tolerability}

As mentioned prior, multiple safety issues exist about the use of TNF inhibitors. The FDA has mandated box warnings for these agents on the increased risk of infections and possible increased risk of malignancies.

\section{Malignancies}

Data suggest that TNF inhibitors may increase the risk of malignancy, especially lymphomas. Although the exact mechanism for this is unknown, it is thought to be due to decreased immune function and immunologic surveillance caused by immunosuppressants. To address this concern, in August 2009 the FDA completed an analysis investigating the risk of malignancies associated with TNF inhibitors. Forty-eight cases of malignancies were identified in children using TNF inhibitors. The majority of these cases were lymphoma but other malignancies such as leukemia, melanoma, renal cell carcinoma, and other solid organ malignancies were observed. ${ }^{11}$

An in-depth review of these cases was published which provided more details. ${ }^{12}$ Of the 48 cases, 31 of the patients were taking infliximab, 15 were taking etanercept, and 2 were taking adalimumab. The 2 patients taking adalimumab had both been previously treated with infliximab which makes causation harder to prove. Over $85 \%$ of patients were on concomitant immunosuppressants. One-half of patients were being treated for inflammatory bowel disease and approximately one-third of the patients were taking a TNF inhibitor for JIA. The rate of reporting of malignancy for patients receiving infliximab was 4 times greater than the background rate of reporting for the general pediatric population. For lymphomas associated with infliximab, the rate of reporting was approximately 18 times that of the background rate. For etanercept, the overall rate of reporting for malignancies was similar to the background rate. However, the rate of lymphoma reporting was 5 times higher than the background rate. ${ }^{12}$ Table 2 provides a further differentiation of the types of malignancies reported.

Another analysis was conducted by the manufacturer of etanercept to investigate the effect of etanercept on malignancies. The analysis included data from 11 years (1998-2009) and patients who received etanercept prior to 18 years of age and developed a malignancy prior to 22 years of age. Based on the data from the randomized controlled 
Table 2 Types of malignancies reported to FDA associated with tumor necrosis factor-alpha inhibitors

\begin{tabular}{lllll}
\hline & Overall & Infliximab & Etanercept & Adalimumab \\
\hline $\begin{array}{l}\text { Hepatosplenic } \\
\text { T cell }\end{array}$ & 10 & 9 & 0 & $\mathrm{I}$ \\
$\begin{array}{l}\text { lymphoma } \\
\text { Hodgkin's }\end{array}$ & 6 & 3 & 2 & $\mathrm{I}$ \\
$\begin{array}{l}\text { lymphoma } \\
\text { Non-Hodgkin's }\end{array}$ & 5 & 5 & 0 & 0 \\
$\begin{array}{l}\text { lymphoma } \\
\text { B-cell }\end{array}$ & 2 & 0 & 2 & 0 \\
$\begin{array}{l}\text { lymphoma } \\
\text { Leukemia }\end{array}$ & 7 & 3 & 4 & 0 \\
$\begin{array}{l}\text { Other } \\
\text { Total }\end{array}$ & 18 & 11 & 7 & 0 \\
\hline
\end{tabular}

trials and extension trials, there were no malignancies reported in the 725 patients treated. However, 18 cases of malignancy were identified in postmarketing and registry data. The types of malignancy included leukemia (4 cases), lymphoma (7 cases), and solid tumors ( 7 cases). The worldwide incidence rate of all malignancies in etanerceptexposed children aged 4 to 17 was 0.02 per 100 patient-years and the estimated US incidence rate was 0.015 per 100 patient-years. The expected malignancy incidence rate of US children aged 4 to 17 is 0.0147 per 100 person-years. Although no statistical assessment was done, the rates appear similar between etanercept-exposed patients and the normal population. However, for lymphoma the etanercept-exposed US incidence rate was 0.01 per 100 patient-years in children aged 4 to 17 years old. The expected US incidence rate for lymphoma in this age group is 0.0026 per 100 person-years. This correlates to a relative risk for developing lymphoma of 3.8 for etanercept-exposed children. The authors noted that most patients were on other concomitant medications, some of which have also been associated with an increased risk of malignancy. ${ }^{13}$

One limitation to these analyses is the comparison to the background rate. There is evidence that patients with autoimmune disorders have a higher incidence of malignancies compared to the general public. In the FDA and in-depth analyses, the comparison for the TNF inhibitor-treated patients was the general pediatric population and not the pediatric population with autoimmune disorders. This could lead to overestimating the risk of the TNF inhibitor therapy in causing malignancies. In addition, these analyses are relying on reporting of these malignancies to the manufacturer or FDA which does not always occur. Therefore, the rate of malignancies may actually be higher than reported in the analysis. Lastly, it is hard to prove that the TNF inhibitor therapy is responsible for the increased risk since these are retrospective case reports.

However, even with these limitations the increased risk of malignancies is still a concern and needs to be considered when starting a child on therapy. Interestingly, the majority of the children were on the TNF therapy for a median of 30 months prior to the malignancy diagnosis. ${ }^{12}$ Based on the data available, it appears that the risk may be greater if treatment is for a longer duration and if used with concomitant immunosuppressants.

\section{Infection}

Similar to other immunosuppressants, TNF inhibitors are associated with an increased risk of infection. As stated in the boxed warning associated with the TNF inhibitors, the risk for infection is higher when taken concomitantly with other immunosuppressant agents. The infections of most concern include tuberculosis, invasive fungal infections, and opportunistic infections. ${ }^{1-5}$

\section{Infliximab: clinical trials}

In a review of trials examining infliximab for inflammatory bowel disease in adolescents, the infection rate was compared to that of adults. In trials including adult patients, the rate of infection was $4 \%$ to $8 \%$ and the infection rate of pediatric patients was $3.9 \%$ with similar types of infections as adults. In one of the analyses reviewed, the rate of herpes zoster in pediatric patients was $3.7 \% .^{14}$

These data are in stark contrast to the percentages from the manufacturer. The package insert states that $56 \%$ of pediatric patients who received infliximab reported an infection compared to $50 \%$ of adults. This discrepancy may be due to the difference in definitions of infections and classification of minor or severe. The majority of infections listed from the manufacturer were upper respiratory tract infections and pharyngitis. Only $6.8 \%$ of patients experienced a serious infection, abscess being the most common. Also, there was a difference in infection rate based on dosing interval. Pediatric patients receiving infliximab every 8 weeks had a much higher rate of infections (74\%) than patients receiving it every 12 weeks $(38 \%) .^{5}$

\section{Etanercept: clinical trials}

In the trial that examined the effect of etanercept on JIA, the rate of upper respiratory tract infections was $35 \%$ during the open labeled portion. In the 2-year extension of the trial, $15 \%$ of patients had to be hospitalized for a serious adverse event, most of which were infectious etiologies. ${ }^{8}$ After the 
4-year extension portion, the rate of serious infections was determined to be 0.04 per patient-year. ${ }^{3}$ In safety data from the 8-year extension trial, there was no increase in the rate of serious adverse events. ${ }^{9}$

Safety of etanercept was evaluated based on data from a registry of pediatric patients treated with etanercept for JIA. This registry examined the safety of etanercept, methotrexate, or the combination of methotrexate and etanercept. For etanercept monotherapy, the rate of medically important infections was 1.8 per 100 patient-years. This is more than was seen in the methotrexate monotherapy group (1.3 per 100 patient-years), but less than in the combination group (2.1 per 100 patient-years). This validates the theory that the risk of infection increases when patients are on multiple immunosuppressants. ${ }^{15}$

In a similar German registry, 322 patients treated with etanercept were evaluated representing 592 patient-years. There were reported infections in 20 of these patients. A variety of infections was observed, including bronchitis, herpex simplex labialis, varicella zoster virus infection, cellulitis, and pneumonia. This represents a $6.2 \%$ risk of infection with treatment. ${ }^{16}$

\section{Adalimumab: clinical trials}

Adalimumab data describe similar infection rates as the other aforementioned TNF inhibitors. In a trial examining the effect of adalimumab on pediatric patients with JIA, the rate for all infections was $45 \%$ within the first 16 weeks of therapy whereas the rate for serious infection was $4 \%$ within 2 years of initiation of therapy. The serious infections included herpes simplex, pneumonia, and herpes zoster. ${ }^{10}$

\section{Case reports}

Since the use of TNF inhibitors has expanded in the pediatric population, many case reports have described infections associated with their use. A case series published in 2010 describes 4 cases of children developing musculoskeletal infections after receiving TNF inhibitors. ${ }^{17}$ All 4 patients were being treated for JIA and each was on TNF inhibitor plus methotrexate. Two patients were treated with etanercept and the other 2 were treated with infliximab with a total range of TNF inhibitor treatment of 6 to 14 months. In all 4 cases, the patients were found to have a musculoskeletal infection with no inciting event. At this medical center in Vancouver, Canada, 31 children were being treated with TNF inhibitors and these cases represent an adverse effect rate of $12.9 \% .^{17}$

In another case report, a patient being treated with infliximab for Crohn's disease developed disseminated cytomegalovirus (CMV) infection. The authors described how the presenting symptoms mimic that of a Crohn's disease flare, making CMV hard to diagnose. Unfortunately in this situation, the disseminated CMV did not respond to antivirals and the infection led to mortality. ${ }^{18}$

Disseminated cutaneous varicella zoster virus infections are described in 2 patients being treated with infliximab for Crohn's disease. Both patients had been treated with infliximab for 2 or more years and were also receiving concomitant immunosuppressant therapy (methotrexate and 6-mercaptopurine). In the first case, the child had received the varicella vaccination and was later exposed to a classmate with varicella infection. Her rash covered most of her lower and upper extremities and she was treated successfully with antiviral therapy. The child in the second case had a history of varicella zoster virus infection 13 years prior and received the varicella vaccination. His rash involved his scalp, face, trunk, and extremities. He too was treated successfully with antivirals but had 2 recurrences. The authors cite infliximab as the likely contributing factor since in both cases their Crohn's disease was well controlled and they had been on long-term infliximab therapy. This case report emphasizes the need for more data on the risk of varicella and other infections in children and determining the best way to diagnose and treat these patients. ${ }^{19}$

A case report of an 8-year-old boy highlights the risk of opportunistic infections in patients treated with TNF inhibitors. This child's Crohn's disease was being treated with infliximab every 5 to 6 weeks for 15 months prior to him developing fevers, cough, anorexia, and night sweats. He was initially treated with a course of azithromycin with some improvement but then subsequent return of symptoms. Once admitted to the hospital, further evaluation was done and cultures of biopsied lymph node revealed Pneumocystis jirovici and Histoplasma capsulatum. After treatment for both infections, the child improved but his histoplasmosis antigen remained elevated. ${ }^{20}$

All of these case reports emphasize the need to consider rarer infectious causes when TNF inhibitor-treated patients present with infectious symptoms. Particular importance should be paid to opportunistic infections and the risk of developing these. In many of these cases, optimal treatment was delayed because these rarer infections were not considered until other therapy failed. Practitioners should consider the potential for these infections when choosing empiric therapy.

\section{Injection-related reactions}

One of the more common adverse effects of TNF inhibitors is injection- or infusion-related reactions. These can range 
from erythema at the injection site to anaphylaxis reactions associated with infusion. The more serious reactions are associated with infliximab since it is not $100 \%$ humanized like the other TNF inhibitors.

When compiling data from 11 evaluations of infliximab use in pediatric patients with inflammatory bowel disease, infusion reactions were observed in $3 \%$ to $39 \%$ of patients or in $0.8 \%$ to $8.6 \%$ of infusions. There appears to be a difference in the rate of reactions in pediatric patients and adult patients when re-treated with episodic doses of infliximab. Children less than 17 years old tend to have less infusion reactions than adults. The infusion reactions associated with infliximab can include hypotension, flushing, fever, nausea, tachycardia, pruritis, fatigue, and chest tightness. Premedication does not appear to be beneficial in preventing these infusion reactions but can be useful for decreasing the chance of another reaction with subsequent infusions. ${ }^{14}$

Reactions associated with etanercept and adalimumab are milder since they do not contain a chimeric portion. In the open label portion of the etanercept study, $39 \%$ of patients experienced an injection-site reaction. ${ }^{8}$ However, in the double blind portion of the study, there was no difference between etanercept and placebo in the rate of injection-site reactions with only 1 patient in each group experiencing a reaction. One possible reason for this is that the reaction is likely a T-lymphocyte mediated delayed-type hypersensitivity. With this type of reaction, tolerance develops with time and the frequency decreases. ${ }^{21}$ If the reaction needs treatment, cold compresses can be applied or topical antihistamine or corticosteroids can be used. In patients treated with adalimumab, $19 \%$ experienced injection-site pain and $16 \%$ experienced an injection-site reaction. In addition, $6 \%$ of patients had a hypersensitivity reaction to adalimumab that was described as a nonserious, localized, allergic rash. ${ }^{1}$

\section{Immunogenicity}

All of the TNF inhibitors have been associated with development of antibodies to the medication. Although these have been measured, the clinical impact is not completely known. For infliximab these antibodies have been associated with increased infusion reactions and loss of effect but for etanercept and adalimumab the clinical impact is not as apparent.

\section{Infliximab}

Human antichimeric antibody (HACA) production is likely the cause of infusion reactions associated with infliximab. A retrospective analysis was done by Miele et al to determine the rate of HACA production in children treated with infliximab. Of the 132 patients treated with infliximab, only 56 had HACA assays measured and of those 22 were inconclusive due to circulated infliximab in the blood. Of the 34 patient samples remaining, 35\% had HACA detected. For the patients who were HACA positive, infusion reactions occurred in a higher proportion of infusions than in patients who were HACA negative (13.8\% vs 3.6\%, respectively, $P<0.01)$. When assessing the degree of HACA production, patients whose concentrations were $8.0 \mu \mathrm{g} / \mathrm{mL}$ or higher were more likely to experience an infusion reaction. This higher concentration of HACA trended to occur in patients whose infliximab infusions were 8 weeks or less apart. Patients who were on concomitant immunosuppressant therapy had a lower risk of HACA development (relative risk: 0.34, $P=0.02$ ) and if HACA developed the concentrations were lower in these patients than in patients not on concomitant therapy. Unfortunately, this analysis did not correlate HACA formation to clinical response. ${ }^{22}$

Another analysis confirmed the incidence rate of the Miele et al analysis. In this review, of the 22 children treated with infliximab, 35.7\% developed HACA. In patients who received induction therapy with only 1 dose, $77.7 \%$ developed HACA compared to only $15.7 \%$ in patients who received 3 induction doses at weeks 0,2 , and $6(P=0.0028)$. The clinical response based on HACA production was assessed in this analysis. Of the 8 children who no longer responded to infliximab therapy, $75 \%$ had positive HACA. This is in contrast to the 14 patients who maintained response in which only $21.4 \%$ were HACA positive. ${ }^{23}$

\section{Etanercept and adalimumab}

In the approval trial for etanercept, only 2 patients of the 25 treated with etanercept developed etanercept antibodies ( $8 \%$ ) and these antibodies were considered non-neutralizing. ${ }^{8}$ No data on the development of these antibodies were reported in the 8-year open label follow-up study. ${ }^{9}$

Adalimumab antibodies were detected in 27 of the 171 patients $(16 \%)$ treated in the approval trial. When comparing patients on concomitant methotrexate or not, the rate for patients on methotrexate was $6 \%$ compared to $26 \%$ for those not receiving methotrexate. ${ }^{10}$

The clinical response rate was not correlated to the antibody production in either study but the percentages were lower than for infliximab probably due to the lack of a chimeric portion. Based on data from these studies and adult trials, the development of antibodies is a concern particularly for infliximab. Concomitant use with another 
immunosuppressant such as methotrexate appears to reduce the risk. Although not commonly measured in clinical practice, antibody production should be considered if a patient starts to lose clinical response from these agents over time.

\section{Autoimmunity}

In addition to the development of antibodies against the agent, an association with other autoantibodies has been described in literature specifically related to etanercept. The production of autoantibodies was assessed in a prospective analysis of 26 children with JIA who were treated with infliximab or etanercept for at least 2 years. Overall, the incidence of developing elevated autoantibodies was $23 \%$ (1/12 patients on infliximab, 5/14 patients on etanercept). Of the 5 patients treated with etanercept who developed autoantibodies, 4 developed anti-R1 antibodies, and 1 developed anti-thyroid antibodies resulting in Hashimoto's thyroiditis. Although measured, the autoantibody production did not affect the patients' JIA clinical course. ${ }^{24}$

Data from a registry evaluating the effect of etanercept on JIA revealed an autoimmune event rate of 5.4 per 100 patient-years. In patients concomitantly treated with methotrexate, this rate decreased to 2.4 per 100 patient-years. The only details about these events were the rates of autoantibody presence: 1.8 per 100 patient-years for etanercept-treated patients and 0.6 per 100 patient-years for patients also on methotrexate. Again, although noted and measured, the clinical effects of these autoimmune antibodies or autoimmune events were not elucidated. ${ }^{15}$

Two case reports have described systemic lupus erythematous development in children treated with etanercept for JIA. In one case, the child developed double-stranded DNA antibodies with an elevation in his ANA titer while being treated with etanercept. He presented with temperature, urticaria, and swollen hands. His symptoms resolved after discontinuing etanercept and initiating steroids. In the other case, the patient developed positive anti-double stranded DNA antibodies with fatigue, hair loss, glomerulonephritis, and severe lung disease. Even with discontinuing etanercept, her disease was not reversible. ${ }^{21}$

Another autoimmune disorder that has been associated with etanercept is inflammatory bowel disease. Interestingly, infliximab is indicated to treat this disease in children whereas etanercept may induce it. Of the 127 patients treated with etanercept for JIA, 5 (3.9\%) developed new onset inflammatory bowel disease. Biopsies from 3 of these patients confirmed aseptic inflammatory bowel disease in 1 patient and Crohn's disease in 2 patients. On discontinuation of etanercept and initiation of infliximab, all patients experienced a complete remission. ${ }^{25}$ Although there are only limited data on this adverse effect, it is one that should be investigated further.

\section{Other adverse effects}

The TNF inhibitors have been associated with a variety of other adverse effects in varying degrees. One of the other serious adverse effects is the increased risk of new onset or exacerbation of demyelinating disorders. To date, there have only been 2 cases reported in patients who had JIA. However, in both cases the patient was 21 years of age at the onset of symptoms. ${ }^{21}$ However, patients with history of these disorders or seizures should not use TNF inhibitors and if a patient on these therapies presents with mental status changes, demyelinating disorders should be on the differential.

Similarly to other immunosuppressants, the TNF inhibitors may cause pancytopenia, leukopenia, neutropenia, aplastic anemia, or thrombocytopenia. Therefore, the blood counts of patients should be monitored while on therapy and therapy should be discontinued if these effects are noted.

Lastly, TNF inhibitors have been associated with reactivation of hepatitis $B$ virus in patients who are chronic carriers of hepatitis B. Although this is a concern, it is less so for children since the majority of children now receive the hepatitis B vaccination starting at birth. However, if a patient has not received the vaccination and has risk factors for hepatitis B infection, they should be screened prior to initiating therapy.

\section{Place in therapy}

The TNF inhibitors offer a treatment option for patients failing treatment with the nonbiologic agents such as methotrexate or azathioprine. In the majority of cases, initiation of TNF inhibitors is associated with a positive clinical response. However due to the limitations of these agents as outlined above, they should not be used as first-line treatment especially in children. New information continues to become available about this class of medications, and with the lack of information in children, the more conservative approach is to reserve the use of these agents for patients with more severe, unresponsive disease.

Because etanercept and adalimumab have the most data supporting their use in pediatric patients, these agents would be preferred over infliximab at this time. Infliximab has the most data to support its use in children with inflammatory bowel disease, whereas, etanercept and adalimumab are indicated for children with JIA. Regardless of the indication 
or age of the patient, the practitioner and patient need to be aware of the risks associated with these medications and make an informed decision, weighing the risks and benefits.

\section{Disclosure}

The author declares no conflicts of interest.

\section{References}

1. Humira [package insert]. North Chicago, IL: Abbott Laboratories; 2009.

2. Cimzia [package insert]. Smyrna, GA: UCB, Inc.; 2010.

3. Enbrel [package insert]. Thousand Oaks, CA: Immunex Corp.; 2008.

4. Simponi [package insert]. Malvern, PA: Centocor Ortho Biotech Inc.; 2010.

5. Remicade [package insert]. Malvern, PA: Centocor Ortho Biotech Inc.; 2009.

6. Tracey D, Klareskog L, Sasso EH, Salfeld JG, Tak PP. Tumor necrosis factor antagonist mechanisms of action: A comprehensive review. Pharmacol Toxicol. 2008;117:244-279.

7. Mpofu S, Fatima F, Moots RJ. Anti-TNF therapies: they are all the same (aren't they?). Rheumatology. 2005;44:271-273.

8. Lovell DJ, Giannini EH, Reiff A, et al. Etanercept in children with polyarticular juvenile rheumatoid arthritis. $N$ Engl J Med. 2000;342: $763-769$.

9. Lovell DJ, Reiff A, Ilowite NT, et al. Safety and efficacy of up to eight years of continuous etanercept therapy in patients with juvenile rheumatoid arthritis. Arthritis Rheum. 2008;58:1496-1504.

10. Lovell DJ, Ruperto N, Goodman S, et al. Adalimumab with or without methotrexate in juvenile rheumatoid arthritis. $N$ Engl J Med. 2008:359: $810-820$.

11. Food and Drug Administration. Information for Healthcare Professionals: Tumor Necrosis Factor (TNF) Blockers (marketed as Remicade, Enbrel, Humira, Cimzia, and Simponi). URL: http:// www.fda.gov/Drugs/DrugSafety/PostmarketDrugSafetyInformation forPatientsandProviders/DrugSafetyInformationforHeathcareProfessionals/ucm 174474.htm

12. Diak P, Siegel J, Grenade LL, Choi L, Lemery S, McMahon A. Tumor necrosis factor alpha blockers and malignancy in children. Arthritis Rheum. 2010;62:2517-2524.
13. McCroskery P, Wallace CA, Lovell DJ, Stryker, et al. Summary of worldwide pediatric malignancies reported after exposure to etanercept. Pediatr Rheumatol [Internet]. 2010 Jun 14 [cited 2010 Nov 8];8:18. http://www.ped-rheum.com/content/8/1/18

14. Veres G, Baldassano RN, Mamula P. Infliximab therapy in children and adolescents with inflammatory bowel disease. Drugs. 2007;67: $1703-1723$.

15. Giannini EH, Ilowite NT, Lovell DJ, et al. Long-term safety and effectiveness of etanercept in children with selected categories of juvenile idiopathic arthritis. Arthritis Rheum. 2009;60:2794-2804.

16. Horneff G, Schmeling H, Biedermann T, et al. The German etanercept registry for treatment of juvenile idiopathic arthritis. Ann Rheum Dis. 2004;63:1638-1644.

17. Morishita K, Petty R, Cairns R, Bolaria R, Cabral D, Turvey S. Serious musculoskeletal infections in children receiving anti-tumor necrosis factor-alpha therapy: a case series. Clin Rheumatol. 2010;29:677-681.

18. Pickering O, Weinstein T, Rubin LG. Fatal disseminated cytomegalovirus infection associated with infliximab and 6-mercaptopurine therapy in a child with Crohn disease. Pediatr Infect Dis J. 2009;28:556.

19. Kunz AN, Rajnik M. Disseminated cutaneous varicella zoster virus infections during infliximab therapy for Crohn's disease: case report of two pediatric patients at one institution. Clin Pediatr. 2010. [Epub ahead of print Sep 13].

20. Tschudy J, Michail S. Disseminated histoplasmosis and pneumocystis pneumonia in a child with Crohn disease receiving infliximab. J Pediatr Gastroenterol Nutr. 2010;51:221-222.

21. Pain CE, McCann LJ. Challenges in the management of juvenile idiopathic arthritis with etanercept. Biologics. 2009;3:127-139.

22. Miele E, Markowitz JE, Mamula P, Baldassano RN. Human antichimeric antibody in children and young adults with inflammatory bowel disease receiving infliximab. J Pediatr Gastroenterol Nutr. 2004;38:502-508.

23. Candon S, Mosca A, Ruemmele F, Goulet O, Chatenoud L, Cezard JP. Clinical and biological consequences of immunization of infliximab in pediatric Crohn's disease. Clin Immunol. 2006;118:11-19.

24. Kanakoudi-Tsakalidou F, Tzimouli V, Pratsidou-Gertsi P, Chronopoulou E, Trachana M. The significance of persistent newly developed autoantibodies in JIA patients under long-term anti-TNF treatment. Cytokine. 2008;42:293-297.

25. Gerloni V, Pontikaki I, Gattinara M, Fantini F. Focus on adverse events of tumour necrosis factor alpha blockade in juvenile idiopathic arthritis in an open monocentric long-term prospective study of 163 patients. Ann Rheum Dis. 2008;67:1145-1152.
Adolescent Health, Medicine and Therapeutics

\section{Publish your work in this journal}

Adolescent Health, Medicine and Therapeutics is an international, peer-reviewed, open access journal focusing on health, pathology, and treatment issues specific to the adolescent age group. All aspects of health maintenance, preventative measures and disease treatment interventions are addressed within the journal and practitioners from

\section{Dovepress}

all disciplines are invited to submit their work as well as healthcare researchers and patient support groups.. The manuscript management system is completely online and includes a very quick and fair peerreview system. Visit http://www.dovepress.com/testimonials.php to read real quotes from published authors. 